\title{
Development of Crohn's Disease in a Child With SLC26A3-related Congenital Chloride Diarrhea: Report of the First Case in East Asia and a Novel Missense Variant
}

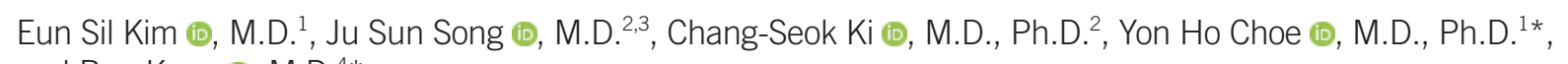
and Ben Kang (iD, M.D. ${ }^{4 *}$

${ }^{1}$ Department of Pediatrics, Samsung Medical Center, Sungkyunkwan University School of Medicine, Seoul, Korea; ${ }^{2}$ GC Genome, Yongin, Korea; ${ }^{3}$ Department of Laboratory Medicine, Green Cross Laboratories, Yongin, Korea; ${ }^{4}$ Department of Pediatrics, School of Medicine, Kyungpook National University, Daegu, Korea

Dear Editor,

Congenital chloride diarrhea (CCD; MIM\#214700) is a rare, autosomal recessive disorder caused by pathogenic variants in the solute carrier family 26 member 3 (SLC26A3; MIM\#126650) gene located on chromosome 7q31. SLC26A3 encodes an apical epithelial $\mathrm{Cl}^{-} / \mathrm{HCO}_{3}{ }^{-}$exchanger located in the ileum and colon [1]. The primary defect of the intestinal $\mathrm{Cl}^{-} / \mathrm{HCO}_{3}{ }^{-}$exchanger, which is coupled with a $\mathrm{Na}^{+} / \mathrm{H}^{+}$exchanger, leads to persistent $\mathrm{Cl}^{-}$rich diarrhea from birth and life-threatening metabolic alkalosis [1]. Recently, a potential association between CCD and inflammatory bowel disease (IBD) has been proposed in Europe [2-4]. However, to date, there have been no reports of IBD development in East Asian patients with CCD. This is the first case of Crohn's disease (CD) developed in a child of East Asian ancestry with genetically confirmed CCD. This study was approved by the Institutional Review Board of Samsung Medical Center (Number 2018-02-007) and was conducted at the Department of Pediatrics, Samsung Medical Center, Seoul, Korea, in 2018. Informed consent was obtained from the patient and his parents.

The patient is a Korean male who was born at $36^{+6}$ weeks of gestation in November 2007. Small bowel dilatation and maternal polyhydramnios were noted on fetal ultrasonography. The patient developed abdominal distension and watery diarrhea at birth, with a rate of 10-12 times per day. Laboratory tests revealed hypochloremic metabolic alkalosis and hypokalemia; the fecal $\mathrm{Cl}^{-}$was elevated to $126 \mathrm{mmol} / \mathrm{L}$. Direct sequencing of the SLC26A3 gene revealed two compound heterozygous variants: a previously reported variant at intron 12 (NM_000111.2:C.1407+3A >C) [5] and a novel missense variant (NM_000111.2:c.2135C > A;p.Thr712Lys),
Received: March 5, 2020

Revision received: May 11, 2020

Accepted: September 11, 2020

Corresponding author: Yon Ho Choe, M.D., Ph.D.

Department of Pediatrics, Samsung Medical Center, Sungkyunkwan University School of Medicine, 81 Irwon-ro, Gangnam-gu, Seoul 06351 , Korea

Tel: +82-2-3410-3527, Fax: +82-2-3410-0043

E-mail: yonho.choe@samsung.com

\section{Co-corresponding author: Ben Kang, M.D.}

Department of Pediatrics, School of Medicine, Kyungpook National

University, 680 Gukchaebosang-ro, Jung-gu, Daegu 41944, Korea

Tel: +82-53-200-2749, Fax: +82-53-200-2029

E-mail: benkang@knu.ac.kr

*These authors contributed equally to this study.

\section{(c) (1) (8)}

\section{(c) Korean Society for Laboratory Medicine}

This is an Open Access article distributed under the terms of the Creative Commons Attribution Non-Commercial License (https://creativecommons.org/licenses/by-nc/4.0) which permits unrestricted non-commercial use, distribution, and reproduction in any medium, provided the original work is properly cited. 
which have not been reported in CCD to date. Genetic testing of the patient's parents revealed that both were heterozygous carriers for the variants (Fig. 1A).

According to the Sequence Interpretation Guidelines of the American College of Medical Genetics and Genomics and the Association for Molecular Pathology [6], the c.1407+3A>C variant could be classified as a likely pathogenic variant (LPV) based on the following evidence: it is absent in the gnomAD population database (PM2), listed as a deleterious mutation in the Human Gene Mutation Database (PP5), and predicted to disturb a consensus splice donor site based on three in silico splice site analysis algorithms (PP3; SpliceSiteFinder, MaxEntScan, and NNSplice) using Alamut Visual v.2.11 (Interactive Biosoftware, Rouen, France). In addition, the PM3 evidence code could be applied to this variant, as the patient reported by Hong, et al. [5] was a compound heterozygote for the founder pathogenic variant (c.2063-1G > T) and the c.1407+3A >C variant. Lastly, the PP4 evidence code could be applied based on the existence of two patients with CCD, including the present patient and the patient reported by Hong, et al. [5].

The c.2135C > A (p.Thr712Ly) variant could also be classified as an LPV based on the following evidence: it is absent in the gnomAD population database (PM2); predicted as a deleterious variant by in silico prediction tools (PP3) using PolyPhen-2 (probably damaging with a score of 1.000), SIFT (deleterious with a score of 0 ), and MutationTaster (disease causing with probability of 1); trans with the $\mathrm{C} .1407+3 \mathrm{~A}>\mathrm{C}$ variant (PM3); and has highly specific patient phenotypes (PP4) [6]. The patient's hypochloremic metabolic alkalosis and hypokalemia were controlled by administration of salt substitution therapy with $\mathrm{NaCl}$ and $\mathrm{KCl}$.

At the age of eight years, the patient developed chronic diar- rhea, abdominal pain, and intermittent fever. Weight loss of $2 \mathrm{~kg}$ over two months was observed without any decrease in growth velocity. Laboratory tests showed a white blood cell count $=8.44 \times 10^{9} / \mathrm{L}$, hemoglobin $=100 \mathrm{~g} / \mathrm{L}$, platelet count $=465 \times 10^{9} / \mathrm{L}$, erythrocyte sedimentation rate $=77 \mathrm{~mm} / \mathrm{hr}$, C-reactive protein $=41.2$ $\mathrm{mg} / \mathrm{L}$, and albumin $=39 \mathrm{~g} / \mathrm{L}$; the other laboratory tests were unremarkable. The fecal immunochemical test was positive, and the fecal calprotectin level was $>1,000 \mu \mathrm{g} / \mathrm{g}$. No pathogens were detected in the stool culture and molecular testing. Ileocolonoscopy revealed multiple aphthous ulcers from the cecum to the rectum (Fig. 1B). The upper gastrointestinal endoscopy findings were unremarkable. Chronic inflammation with cryptitis was observed by histology. Magnetic resonance enterography revealed enhanced wall thickening in the distal descending colon, sigmoid colon, and rectum, without evidence of structuring or penetrating complications. Pelvic magnetic resonance imaging showed perianal inflammation at the 3 and 12 o'clock direction. The patient was diagnosed as having CD with an Ala, L2, B1p, G0 phenotype according to the Paris classification [7]. The patient's pediatric $C D$ activity index score was 30 , and simple endoscopic score for $C D$ was 18.

The mechanism underlying the association between CCD and IBD has yet to be elucidated. A previous study has shown that pathogenic variants of SLC26A3 are associated with a lack of a firmly adherent mucus layer and barrier impairment in mice [8]. In addition, intestinal SLC26A3 mRNA expression has been shown to be downregulated both in patients with ulcerative colitis and animal models [9]. Recently, it has been reported that tumor necrosis factor- $\alpha$ acts reciprocally with SLC26A3 and reduces its expression, leading to intestinal inflammation [10]. Further studies are required to reveal the underlying mechanism responsible for the development of $C D$ in $C C D$ patients.
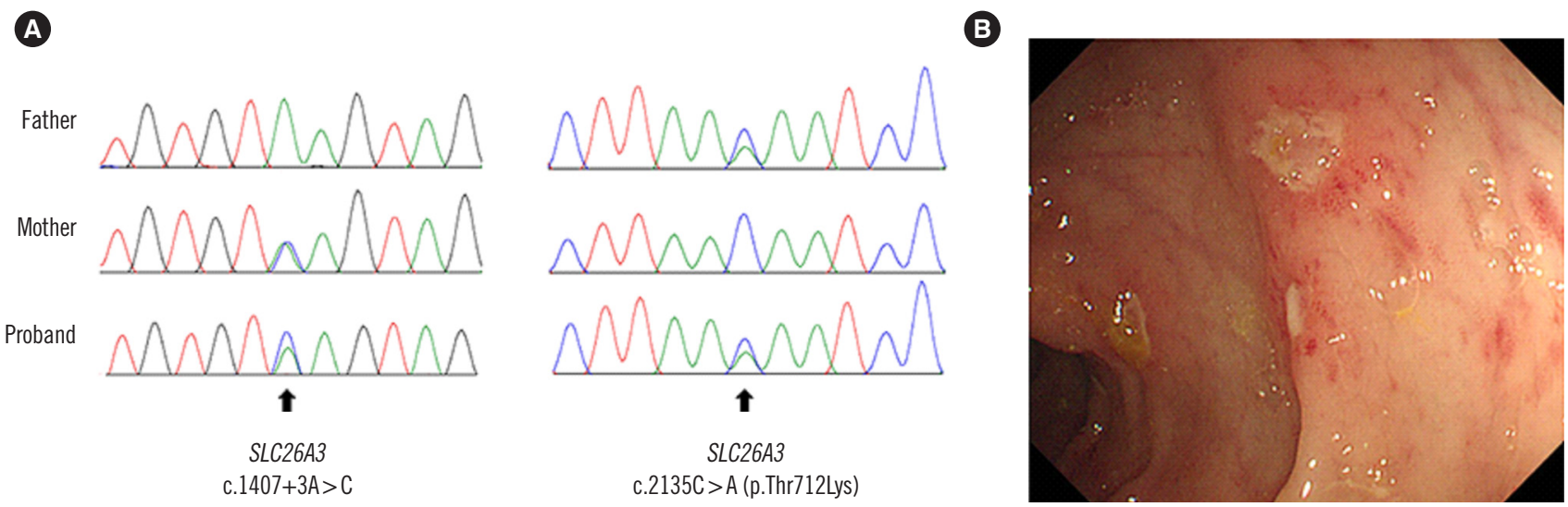

Fig. 1. Diagnosis of congenital chloride diarrhea and Crohn's disease. (A) Sanger sequencing of the SLC26A3 gene in the patient and his parents. (B) Ileocolonoscopy at the diagnosis of Crohn's disease. 
In conclusion, we report the first case of $\mathrm{CD}$ development in a child with SLC26A3-related CCD in East Asia, who also carried a novel missense variant of the SLC26A3 gene. We insist that the development of IBD should be closely monitored in patients with underlying $C C D$.

\section{ACKNOWLEDGEMENT}

None.

\section{AUTHOR CONTRIBUTIONS}

ESK contributed to data acquisition, analysis, and interpretation and drafting of the initial manuscript. JSS contributed to data analysis and interpretation and drafting of the initial manuscript. C-SK contributed to data analysis and interpretation and critical revision for important intellectual content. YHC contributed to the conception and design of the study, data acquisition, and critical revision for important intellectual content. BK contributed to the conception and design of the study; data acquisition, analysis, and interpretation; and critical revision for important intellectual content. All authors approved the final version of the article.

\section{CONFLICTS OF INTEREST}

No potential conflicts of interest relevant to this study are reported.

\section{RESEARCH FUNDING}

This work was supported by the National Research Foundation of Korea (NRF) grant funded by the Korean government (MSIT) (No. 2017R1C1B5076980).

\section{ORCID}

$\begin{array}{ll}\text { Eun Sil Kim } & \text { https://orcid.org/0000-0003-2012-9867 } \\ \text { Ju Sun Song } & \text { https://orcid.org/0000-0003-4607-872X } \\ \text { Chang-Seok Ki } & \text { https://orcid.org/0000-0001-7679-8731 } \\ \text { Yon Ho Choe } & \text { https://orcid.org/0000-0003-1525-7688 } \\ \text { Ben Kang } & \text { https://orcid.org/0000-0002-8516-9803 }\end{array}$

\section{REFERENCES}

1. Wedenoja S, Pekansaari E, Höglund P, Mäkelä S, Holmberg C, Kere J. Update on SLC26A3 mutations in congenital chloride diarrhea. Hum Mutat 2011;32:715-22.

2. Hihnala S, Höglund P, Lammi L, Kokkonen J, Ormälä T, Holmberg C. Long-term clinical outcome in patients with congenital chloride diarrhea. J Pediatr Gastroenterol Nutr 2006;42:369-75.

3. Harris RE, Tayler R, Russell RK. Congenital chloride-losing diarrhoea and Crohn's disease: a diagnostic and therapeutic challenge. Frontline Gastroenterology. Epub ahead of print. doi:10.1136/flgastro-2019-101283

4. Norsa L, Pigneur B, Goulet O, Talbotec C, Lengline H, Rummele F. Potential association between congenital chloride diarrhea and inflammatory bowel disease. Dig Liver Dis 2016;48:e268.

5. Hong J, Seo JK, Ko JS, Cheong HI, Choi JH, Lee JH, et al. Congenital chloride diarrhea in Korean children: novel mutations and genetic characteristics. Eur J Pediatr 2013;172:545-50.

6. Richards S, Aziz N, Bale S, Bick D, Das S, Gastier-Foster J, et al. Standards and guidelines for the interpretation of sequence variants: a joint consensus recommendation of the American College of Medical Genetics and Genomics and the Association for Molecular Pathology. Genet Med 2015;17:405-24.

7. Levine A, Griffiths A, Markowitz J, Wilson DC, Turner D, Russell RK, et al. Pediatric modification of the Montreal classification for inflammatory bowel disease: the Paris classification. Inflamm Bowel Dis 2011;17: 1314-21.

8. Xiao F, Yu Q, Li J, Johansson ME, Singh AK, Xia W, et al. Slc26a3 deficiency is associated with loss of colonic HCO3- secretion, absence of a firm mucus layer and barrier impairment in mice. Acta Physiol (Oxf) 2014;211:161-75.

9. Yang H, Jiang W, Furth EE, Wen X, Katz JP, Sellon RK, et al. Intestinal inflammation reduces expression of DRA, a transporter responsible for congenital chloride diarrhea. Am J Physiol 1998;275:G1445-53.

10. Ding X, Li D, Li M, Tian D, Yu H, Yu Q. Tumor necrosis factor- $\alpha$ acts reciprocally with solute carrier family 26 , member 3 , (downregulated-inadenoma) and reduces its expression, leading to intestinal inflammation. Int J Mol Med 2018;41:1224-32. 